〔Med. Entomol. Zool. Vol. 54 No. 1 p. $43-50$ 2003〕

\title{
A laboratory oviposition study in Aedes albopictus (Diptera: Culicidae) with reference to habitat size, leaf litter and their interactions.
}

\author{
Hamady DienG ${ }^{1)}$, Michael Boots ${ }^{2)}$, Yoshio Tsuda ${ }^{1)}$ and Masahiro TAKAGI ${ }^{1)}$ \\ 1) Department of Vector Ecology and Environment, Institute of Tropical Medicine, \\ Sakamoto 1-12-4, Nagasaki 852-8523, Japan \\ 2) Department of Biological Sciences, Stirling University, Stirling, FK9 4LA 32962, Scotland
}

(Received: 13 August 2002; Accepted: 20 November 2002)

\begin{abstract}
This work presents the results of an experiment on the oviposition of Aedes albopictus (Skuse) where the oviposition sites were different sized-containers with or without leaf from the maple (Acer buergerianum) or from the camphorous (Cinnamomum japonicum). Oviposition responses varied according to container type, to leaf presence/absence as well as leaf type and amount. Oviposition was highest in the "large" container and in the presence of leaves, especially maple leaves, known as good larval nutritional substrate. In nature, container opening surface size is often correlated with the permanence of water as well as leaf litter input; thus, the most likely explanation of our results is that Ae. albopictus can discriminate habitats for oviposition that increase success for its offspring.
\end{abstract}

Key words: Aedes albopictus, oviposition, habitat size, leaf litter, interactions

\section{INTRODUCTION}

Oviposition site selection is an essential part of the life history of all mosquitoes because it is a critical factor in both the survival and the population dynamics (Bentley and Day, 1989). Number of studies has dealt with this topic and has shown that overall, chemical and physical aspects of the site and their interactions greatly influence oviposition behavior.

In some cases, such behavior in combination with anthropic activities has resulted in an important public health problem. To date, one of the most attractive cases is Aedes albopictus (Skuse). The recent introduction of this mosquito from Southeast Asia to non-indigenous countries (Reiter, 1998) is a direct consequence of its oviposition behavior. Females lay eggs not only in natural containers but also in artificial containers (Makiya, 1968; Eshita and Kurihara, 1979; Hawley, 1988) and prefer cry- ptic microhabitats (Hawley, 1988). To date it has become well established in the Western Hemisphere and is being considered second to $A e$. aegypti (Linnaeus) in its importance as vector of dengue and dengue hemorrhagic fever (Hawley, 1988; Knudsen et al., 1996).

Tsuda et al. (1994) have shown that habitat size influences prevalence of Ae. albopictus. They found that "open type" tree hole was more preferred than the "closed type" and suggested that such an attractiveness is related to potential of the "open type" to collect more water and leaf litter falls. Laboratory (Barrera, 1996) and field (Juliano, 1998) investigations reported that Ae. albopictus outperformed Ae. aegypti under litter-based resources. Recently, Lounibos et al. (2002) have reported that performance of Ae. albopictus is particularly sensitive to the presence/absence of leaf litter. With regard to these reports, it seems likely, therefore, that this mosquito in nature is largely determined by con- 
tainer habitat characteristics including size and leaf litter content.

Although a few experimental studies on Ae. albopictus oviposition have used diverse container types (Yan et al., 1995), they did not focus on the container size/ its opening surface size and the related leaf litter input. So, there is little analysis of the significance of these properties on Ae. albopictus oviposition behavior. Thus, experiments incorporating both container size and leaf litter may provide some insight into the habitats-colonization properties of this mosquito.

We conducted experiments on Ae. albopictus oviposition behavior with special reference to container size and leaf litter. We examined the effect of two sized containers, large and small and leaf litter quantity and quality in the oviposition preference of $A$ e. albopictus. In this paper we show that these two containers as well as leaf litter type and amount have different attractiveness.

\section{Materials and Methods}

\section{Experimental features}

The Ae. albopictus used in this study was collected as adults in 1998 in Nagasaki, Japan and has since been maintained at $23-25^{\circ} \mathrm{C}, 60-80 \% \mathrm{RH}$, and $\mathrm{L}: \mathrm{D} 12: 10$ (with 2 hours as dusk) in the laboratory. Larvae were reared based on Tsuda et al. (1991) methods. Adults were provided sucrose solution (3\%) and blood meal from mice was provided to females 4 to 5 days after emergence. In all experiments, 10 males (to insure insemination) and 20 females, 3 days after being engorged on mouse were placed in each cage.

The oviposition cages were cubic $(20 \times$ $20 \times 20 \mathrm{~cm}$ ) and consisted of metal wire and mesh. At the bottom center of the cage was placed a circular (surface $=100$ $\mathrm{cm}^{2}$ ) amber plastic on which four discs were delimited and painted black so as each disc was approximately at equal distance from the nearest neighbor (Fig. 1). On these discs were placed the oviposition cups which consisted of $8.5 \times 5.5 \mathrm{~cm}$ (large) and $3.5 \times 5.5 \mathrm{~cm}$ (small) plastic containers. Each was lined with a filter paper and half-filled with tap water stored in container in the laboratory for at least a week.

Leaves of maple (Acer buergerianum) and of cinnamon (Cinnamomum japonicum) (both of which plants were quite common in Nagasaki) used in this study were collected at roughly equal amounts from both trees and the ground, cleaned of debris, stored dry, and their petioles removed. Maple is a deciduous tree of which leaves are 4 to $8 \mathrm{~cm}$ long and lightly colored. Cinnamon is a dense broad-leaved evergreen tree of which leaves are oval, dark green on the upper lustrous surface, paler green underneath and hard. The fresh as well as the dried leaves are highly aromatic. These different leaves have been shown to have differential effects on Ae. albopictus larval development with maple providing better growth (Dieng et al., 2002). In all experiments with leaves, we have used $0.5 \mathrm{~g}$ per container as low amount and $2.0 \mathrm{~g}$ per container as high amount.

\section{Bioassays}

As oviposition peaks 3 days after blood meal in Ae. albopictus (Tsuda, pers. com.), the experimental batches were placed into the experimental cage. A replicate corresponded to one positioning of the 4 containers on the circular amber plastic (Fig. $1)$.

\section{Experiment 1}

Container size effect on oviposition of Ae. albopictus

To determine whether there would be a preference for a sized container, three different combinations with "large" and "small" containers were made: $[3$ large +1 small], [3 small +1 large] and [2 large +2 small]. In each case, the 4 containers were placed in cage as shown in Fig. 1. 


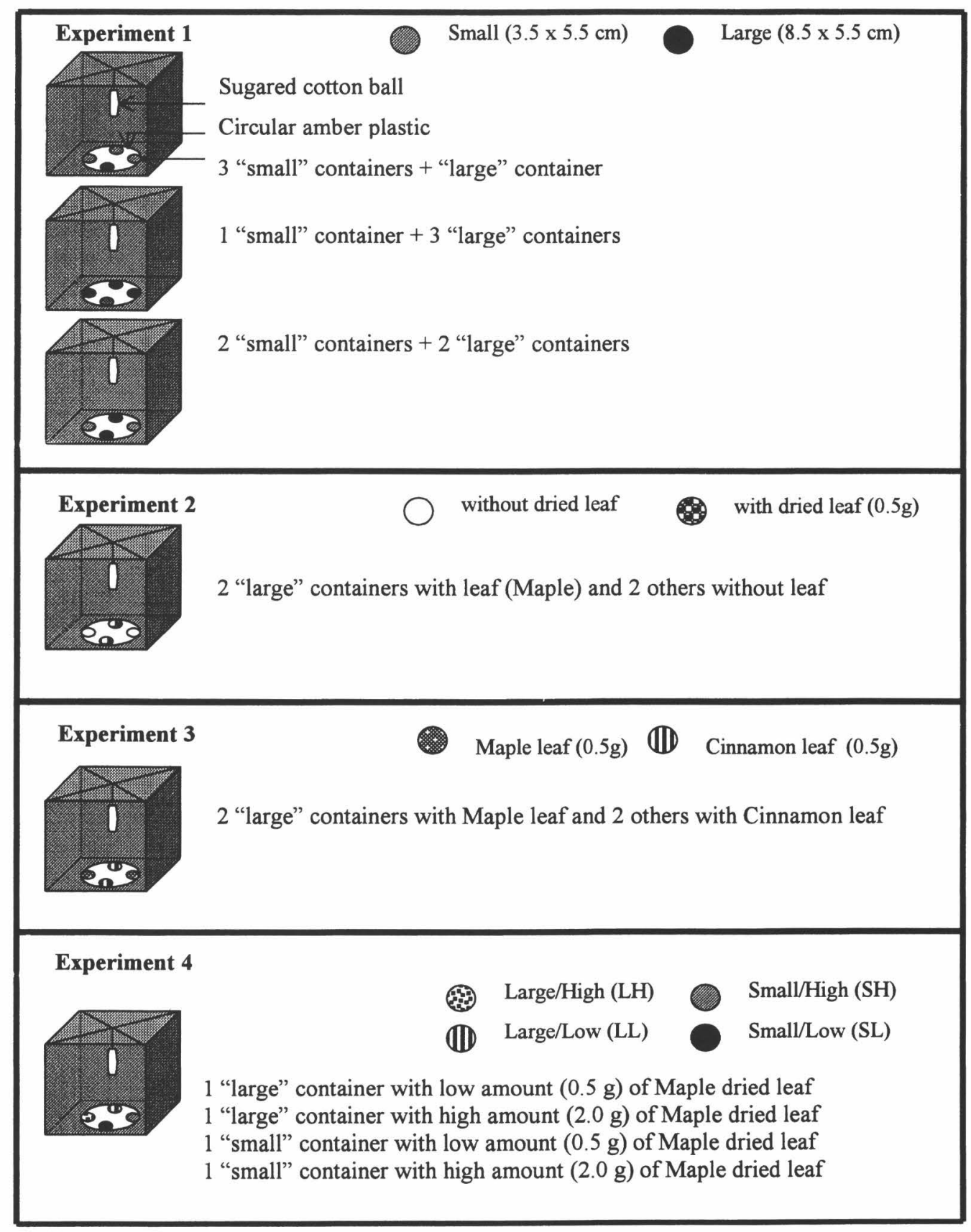

Fig. 1. Experimental design.

Experiment 2

Leaves presence effect on oviposition of Ae. albopictus

To determine whether leaf influences the selection of oviposition sites, we added $0.5 \mathrm{~g}$ of dry maple in 2 large containers and only tap water in the two remaining ones.

\section{Experiment 3}

Leaves type effect on oviposition of Ae. albopictus 
As there is a possibility that leaves are influential to ovipositing females $A e$. albopictus, we targeted also to find out whether the type of leaf will influence such females. For that purpose, we have added $0.5 \mathrm{~g}$ of dry maple leaves in 2 large containers and the amount but of cinnamon added in the two remaining ones.

\section{Experiment 4}

Container size and leaf amount interactions effect on oviposition of Ae. albopictus

To know whether there would be a pref- erence for a container size and an associated amount leaf litter, four different combinations using the two sized-containers and 2 amount of maple dry leaf (low and high) were made: ["large"/high], ["large"/ low], ["small"/high], and ["small"/low]. The 4 containers of each combination were placed on the circular amber plastic so that a large container has as neighbor a small container.

Data collection and statistical analysis

In all experiments, oviposition period
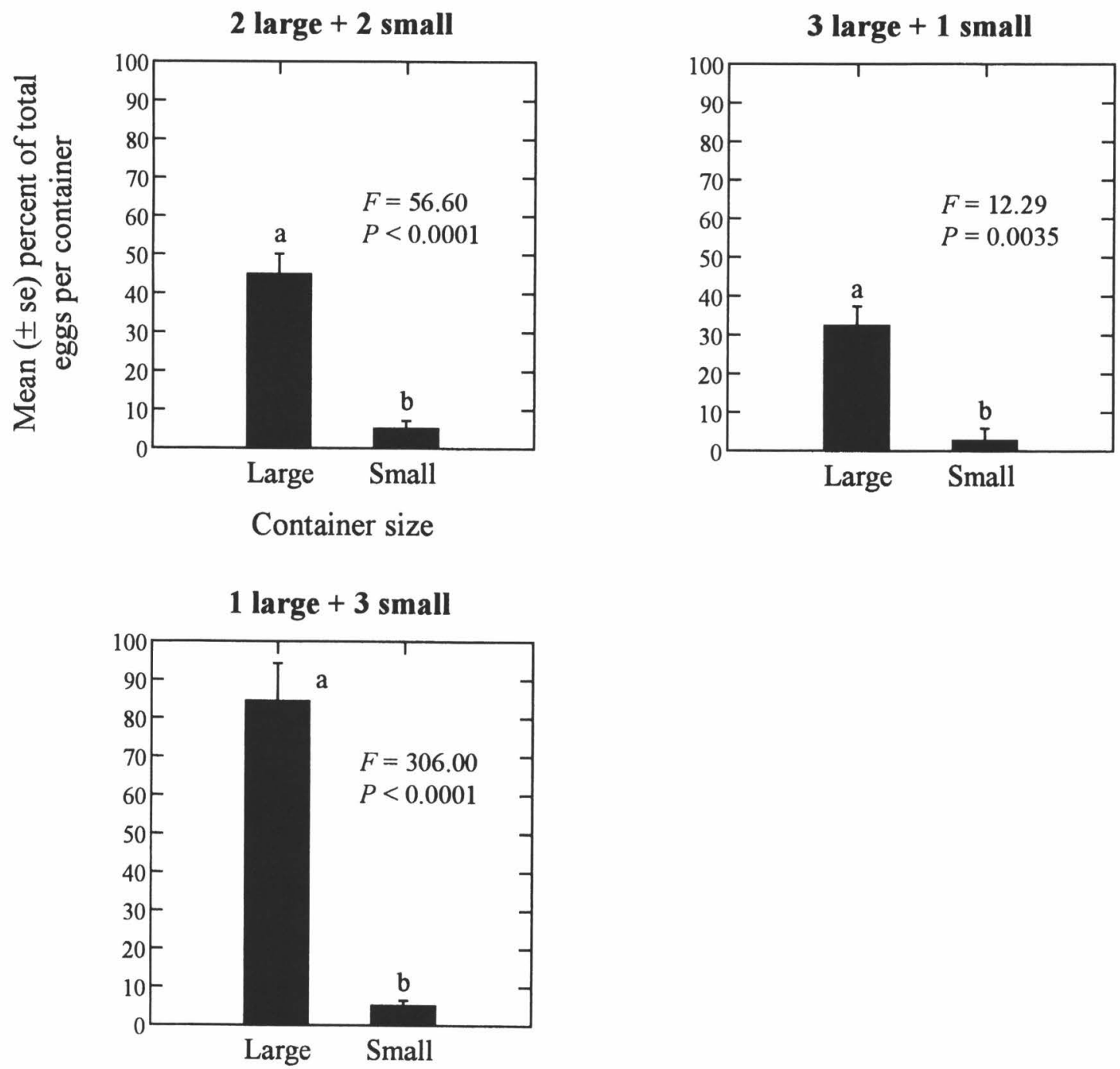

Fig. 2. Effect of container size on selection of oviposition sites by Ae. albopictus [by Anova bars with the same letter do not show a significant difference $(P<0.05)]$. 
was three days after the start of the experiment. The counting of the number of eggs deposited on filter papers and those floating as well as those on the edges of the containers scored bioassays. These numbers were converted into percentages and analyzed by Anova using the SYSTAT statistical software package (Wilkinson, 1996). Means in the fourth experiment were separated with Tukey HSD test.

\section{RESULTS}

\section{Experiment 1}

Container size effect on oviposition of Ae. albopictus

Container size affected significantly oviposition responses of Ae. albopictus (Fig. 2 ). When there were 3 large and 1 small containers, larger containers were significantly more preferred by Ae. albopictus: $31.9 \pm 4.9 \%$ (mean) of $406.7 \pm 70.9$ total eggs were deposited in large containers versus $2.7 \pm 2.7 \%$ in the small containers. A similar pattern was observed when there was more "small" containers than "large" ones. When there was an equal number of both sizes of containers, the "large" one elicited significantly more ovipositions. It is likely that the container with a "large" opening size attracts more ovipositing Ae. albopictus than does the "small" one (Fig. 2).

\section{Experiment 2}

Effects of leaf presence effect on oviposition of Ae. albopictus

The containers with leaves were significantly more preferred by $A e$. albopictus as oviposition sites (Fig. 3). $38.2 \pm 3.1 \%$ (mean) of total eggs $(774.3 \pm 115.2)$ were deposited in containers with leaf versus $11.8 \pm 2.3 \%$ in those without leaf.

\section{Experiment 3}

Effects of leaf type effect on oviposition of Ae. albopictus

Ae. albopictus females tended to prefer more containers with maple as oviposition sites by depositing $28.9 \pm 3.7 \%$ (mean) of total eggs $(690.1 \pm 74.5)$ in such containers versus $21.1 \pm 1.8 \%$ in those with cinnamon leaf (Fig. 3). However there was no a significant difference between these two mean percentages (Fig. 3).

\section{Experiment 4}

Co-effects of container-size and leaf amount on oviposition of Ae. albopictus

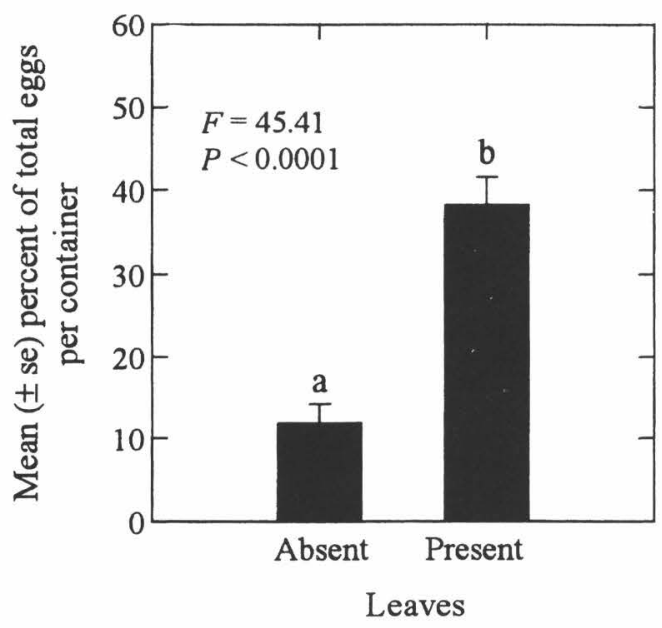

Fig. 3. Effect of leaf litter on selection of oviposition sites by $A e$ albopictus [by Anova bars with the same letter do not show a significant difference $(P<0.05)]$.

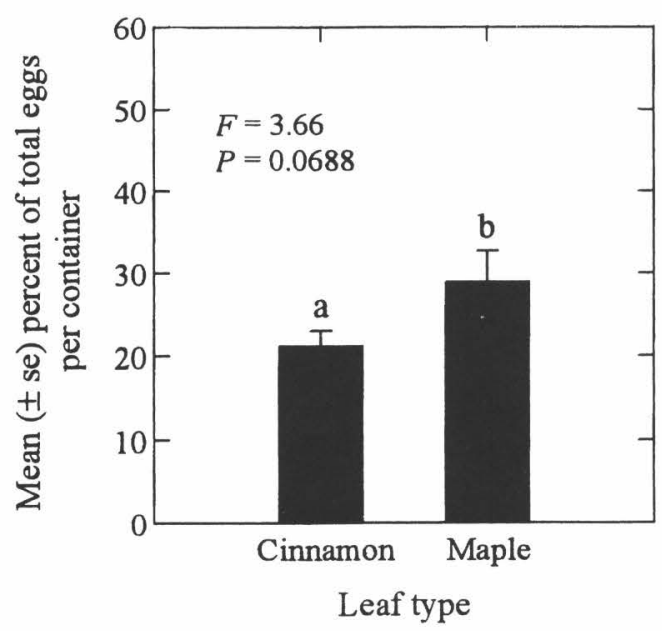

Fig. 4. Effect of leaf litter type on selection of oviposition sites by Ae. albopictus [by Anova bars with the same letter do not show a significant difference $(P<0.05)]$. 


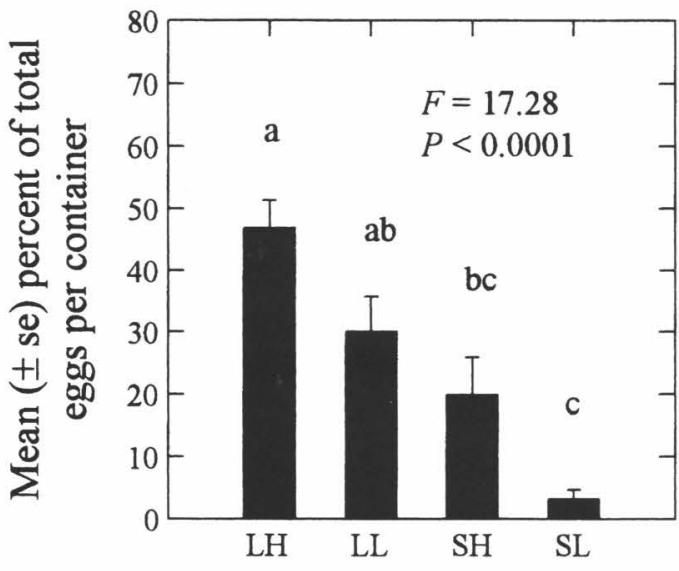

\section{Container and leaf amount}

Fig. 5. Effect of the interactions container size and leaf litter amount on selection of oviposition sites by Ae. albopictus [by Anova bars with the same letter do not show a significant difference $(P<0.05)]$.

For the different treatments, there was steadily decreasing egg deposition when progressing from large container with high leaf amount to large with low leaf amount to small container with high leaf amount to small with low leaf amount. However no significant differences in egg deposition were observed between the treatment except for large container with high leaf amount and small container with low leaf amount (Fig. 4).

In the large containers, there was a tendency that more eggs were deposited in those with high leaf amount than in those with a low amount. The pattern in the small containers was similar, with the container with high leaf amount showing numerically more egg deposition (Fig. 5).

\section{Discussion}

Bradshaw and Holzapfel (1983) reported that most container-breeding mosquitoes prefer drought-resistant containers. In Japan, Tsuda et al. (1994) observed a higher prevalence of Ae. albopictus in tree holes with large opening surface and volume than in those with small dimensions. In general, mosquito larvae, Aedes in particular, feed on decaying leaf litters (Fish and Carpenter, 1982) that are the source of all nutrients they need for development (Kitching, 1983). According to Walker and Merrit (1988), the degree to which these leaves fall in natural or artificial containers is largely dependent on the container dimensions. Indeed containers with large top-opening surface receive more leaf litter than the small ones (Tsuda et al., 1994).

In addition, there is a positive correlation between container size and the permanence of water medium (Bradshaw and Holzapfel, 1983). Indeed, small containers tend to dry or to overflow more rapidly than the large ones. As such, this rapid event of drying and overflowing has direct detrimental effects on Ae. albopictus immatures. Overflow contributes much to the impoverishment in resource by removing most nutrients. In a drying container, the decreasing of resource quality (Aspbury and Juliano, 1998) with increasing larval density enhance intraspecific competition and larval mortality (Alto and Juliano, 2001). With reference to these reports, it is likely that ovipositing Ae. albopictus in the present study has chosen the large container since it has potentially higher nutrient resources for its offspring and low desiccation/overflow risks.

Ae. albopictus preferred containers with leaves, especially maple leaves to either those with cinnamon or those without leaves. These leaves have been demonstrated to have differential effects on the development of insects. Indeed, Ae. albopictus (Dieng et al., 2002) and Ae. triseriatus (Say) (Fish and Carpenter, 1982) larvae grew considerably fast and attained a large size when supplied with maple leaf litter. Lederhouse et al. (1992) showed that leaves of Cinnamomum camphor, a species related to the cinnamon used here, were toxic to two lepidoptera caterpillars that used these leaves as food. Aside from toxicity effects, some other compounds of cinnamon also had function as feeding inhibitors. Dieng et al. (2002) demonstrated 
that when cinnamon senescent leaves were the nutritional substrate of $A$ e. albopictus, larval developmental was delayed, mortality was high and resulted in smallsized adults. Ward and Cummins (1979) considered leaves inducing a good growth to be high quality food and those limiting development to be poor quality food. Thus, it appears that Ae. albopictus females can respond to greater food resource patches for its offspring. Such a behavior aims to lessen intraspecific competition as well as a survival mechanism since food has a positive effect on immature survival, duration of development, and size at emergence (Mori, 1979; Lord, 1998).

Because we conducted our experiments under laboratory conditions, the biological implications of the results obtained here are not clear. In particular, the scale at which adults modify their choice of oviposition sites is not known.

Although bioassays were conducted with specimens that have been maintained in the laboratory for a long period of time, ovipositing females had clearly discriminated between "large" and "small" containers, and between containers with leaves and those without leaves. With regard to previous studies (Fish and Carpenter, 1982; Bradshaw and Holzapfel, 1983; Walker and Merrit, 1988; Lederhouse et al., 1992; Tsuda et al., 1994; Aspbury and Juliano, 1998; Alto and Juliano, 2001; Dieng et al., 2002), these behaviors may not be a result of adaptation to colonization.

At the very least, our results suggest that $A e$ albopictus can assess the quality of potential breeding sites with respect to suitability for larval development completion. Additional investigations are needed to clarify the extent to which container size and detritus substrates interact and its ultimate effect of the dynamic of container-breeding mosquitoes, $A$ e. albopictus in particular.

\section{ACKNOWLEDGEMENTS}

We thank Mayumi Dieng, Hio Dieng, Masako Koura, and Emiko Urakawa for their direct or indirect assistance.

\section{REFERENCES}

Alto, B. W. and Juliano, S. A. 2001. Precipitation and temperature effects on populations of Aedes albopictus (Diptera: Culicidae): implications for range expansion. J. Med. Entomol., 38: 646-656.

Aspbury, A. S. and Juliano, S. A. 1998. Negative effects of habitat drying and prior exploitation on the detritus resource in an ephemeral aquatic habitat. Oecologia (Berl.), 115: 137-148.

Barrera, R. 1996. Competition and resistance to starvation in larvae of container-inhabiting Aedes mosquitoes. Ecol. Entomol., 21: 117-127.

Bentley, M. D. and Day, J. F. 1989. Chemical ecology and behavioral aspects of mosquito oviposition. Annu. Rev. Entomol., 34: 401-421.

Bradshaw, W. E. and Holzapfel, C. M. 1983. Predatormediated, non equilibrium coexistence of tree-hole mosquitoes in southern Northern America. Oecologia, 57: 239-257.

Dieng, H., Mwandawiro, C., Boots, M., Morales, R., Satho, T., Tsuno, N., Tsuda, Y. and Takagi, M. 2002. Leaf litter decay process and the growth performance of Aedes albopictus larvae (Diptera: Culicidae). J. Vector Ecol., 27: 31-38.

Eshita, Y., and Kurihara, T. 1979. Studies on the habitats of Aedes albopictus and Aedes riversi in the Southwestern part of Japan. Jpn. J. Sanit. Zool., 30: 181-186 (In Japanese with English summary).

Fish, D. and Carpenter, R. S. 1982. Leaf litter and larval mosquito dynamics in tree-hole ecosystems. Ecology, 63: 283-288.

Hawley, W. A. 1988. The biology of Aedes albopictus. J. Am. Mosq. Control Assoc. (Suppl.), 1: 1-40.

Juliano, S. A. 1998. Species introduction and replacement among mosquitoes: Interspecific resource competition or apparent competition? Ecology, 79: 255-268.

Kitching, R. L. 1983. Community structure in waterfilled treeholes in Europe and Australiacomparisons and speculations. In Phytotelmata: terrestrial plants as hosts for aquatic insect communities (ed. Frank, J. H. and Lounibos, L. P.), pp. 
205-222, Plexus, Medford, NJ.

Knudsen, A. B., Romi, R. and Majori, G. 1996. Occurrence and spread in Italy of Aedes albopictus, with implications for its introduction into other parts of Europe. J. Am. Mosq. Control Assoc., 12: 177-183.

Lederhouse, R. C., Matthew, P. A., Nitao, K. N. and Mark, S. 1992. Differential use of lauraceous hosts by swallowtail butterflies, Papilio troilus and $P$. palamedes (Papilionidae). Oikos, 63: 244-252.

Lord, C. C. 1998. Density dependence in larval Aedes albopictus (Diptera: Culicidae). J. Med. Entomol., 35: 825-829.

Lounibos, L. P., Suarez, S., Menendez, Z., Nishimura, N., Escher, L., O'Cornell, S. M. and Rey, J. R. 2002. Does temperature affect the outcome of larval competition between Aedes aegypti and Aedes albopictus? J. Vector Ecol., 27: 85-95.

Makiya, K. 1968. Population dynamics of larvae overwintering in southern Japan. Jpn. J. Sanit. Zool., 19: 223-229 (In Japanese with English summary).

Mori, A. 1979. Effects of larval density and nutrition on some immature and adults Aedes albopictus. Trop. Med., 21: 85-103.

Reiter, P. 1998. Aedes albopictus and the world trade in used tires, 1985-1995: the shape of things to come? J. Am. Mosq. Control Assoc., 14: 83-94.

Tsuda, Y., Takagi, M. and Wada, Y. 1991. Preliminary laboratory study on population growth of Aedes albopictus. Trop. Med., 33: 41-46.

Tsuda, Y., Takagi, M. and Wada, Y. 1994. Ecological study on mosquito community in tree holes in Nagasaki, Japan, with reference to Aedes albopictus (Diptera; Culicidae). Jpn. J. Sanit. Zool., 45: 103-111. Walker, E. D. and Merrit, R. W. 1988. The significance of leaf detritus to mosquito (Diptera: Culicidae) productivity from treeholes. Environ. Entomol., 17: 199-206.

Ward, G. M. and Cummins, K. W. 1979. Effects of food quality on growth of a stream detritivore, Paratendipes albimanus (Meigen) (Diptera: Chironomidae). Ecology, 60: 57-64.

Wilkinson, L. (1996) Systat 7.0 for windows: Statistics. SPSS Inc., $751 \mathrm{pp}$.

Wilton, D. P. 1968. Oviposition site selection by the tree hole mosquito, Aedes triseriatus (Say). J. Med. Entomol., 5: 189-194.

Yan, H. H., Lee, C. Y., Chong, N. L., Foo, A. E. and Lim, M. P. 1995. Oviposition site preference of Aedes albopictus in the laboratory. J. Am. Mosq. Control Assoc., 11: 128-132. 